\title{
Depresión perinatal en tiempos del COVID-19: rol de las redes sociales en Internet
} Perinatal depression in times of COVID-19: the role of social media on the Internet

\section{Correspondencia Elizabeth Espinoza-Portilla espinoza.portilla@gmail.com}

Recibido: 22/03/2020 Arbitrado por pares Aprobado: 30/03/2020

Citar como: Bermejo-Sánchez $F R$ Peña-Ayudante WR, EspinozaPortilla E. Depresión perinatal en tiempos del COVID-19: rol de las redes sociales en Internet. Acta Med Peru. 2020;37(1):88-93. doi: https://doi.org/10.35663/ amp.2020.371.913
Fredy R. Bermejo-Sánchez ${ }^{1, a}$, William R. Peña-Ayudante ${ }^{1, b}$, Elizabeth Espinoza-Portilla $a^{2, c}$

Universidad Nacional José Faustino Sánchez Carrión. Huacho, Perú.

Universidad ESAN. Lima, Perú.

Médico cirujano especialista en Psiquiatría, doctor en Salud Pública; ${ }^{b}$ médico cirujano especialista en Ginecología y Obstetricia, doctor en Salud Pública; ${ }^{\mathrm{c}}$ médico cirujano; doctora en Gobierno y Políticas Públicas.

\section{RESUMEN}

Las emergencias en salud pública como la pandemia del COVID-19 podrían llevar a situaciones de estrés extremo, ansiedad y depresión en mujeres en etapa perinatal. La depresión perinatal puede ocasionar a la madre, al recién nacido y al entorno familiar múltiples consecuencias negativas. Las redes sociales, bien utilizadas, pueden constituir una herramienta valiosa de apoyo para las mujeres en esta etapa de su vida. Este artículo describe el rol de las redes sociales en Internet como soporte de las gestantes y madres con depresión, particularmente en emergencias de salud pública a nivel mundial como la pandemia del COVID-19. Las redes sociales pueden contribuir a la salud y al bienestar al facilitar la interacción social, construir comunidades, normalizar el comportamiento de búsqueda de ayuda, inspirar cambios saludables en el estilo de vida y ayudar a la investigación médica. Es fundamental educar adecuadamente a las mujeres en etapa perinatal en alfabetización digital en salud que incluya el uso eficiente de las redes en Internet como soporte socioemocional y la evaluación de la calidad de información que se encuentra en las redes sociales y en Internet para una adecuada toma de decisiones.

Palabras clave: Depresión posparto; Infecciones por coronavirus; Apoyo social; Medios de comunicación sociales (fuente: DeCS BIREME).

\begin{abstract}
Public health emergencies such as COVID-19 pandemic could lead to situations of extreme stress, anxiety and depression in women in the perinatal period. Perinatal depression may lead to multiple negative consequences for mothers, newborns, and their relatives. Appropriate use of social media may be a valuable supporting tool for women during this stage of their lives. This paper describes the role of social media on the Internet for supporting pregnant women and mothers with depression, particularly in global public health emergencies such as the COVID-19 pandemic. Social media may contribute to support health and wellness by facilitating social interaction, building communities, normalizing help-seeking behavior, inspiring healthy lifestyle changes, and promoting medical research. It is essential to adequately educate women in the perinatal stage in digital health literacy including the efficient use of social media for socio-emotional support and assuring the quality of the information circulating in social media and in Internet for proper decision-making.
\end{abstract}

Keywords: Depression; Postpartum; Coronavirus infections; Social support; Social media (source: MeSH NLM). 


\section{INTRODUCCIÓN}

Si bien el nacimiento de un niño es a menudo recibida con mucha alegría y entusiasmo, puede generar preocupación en los nuevos padres, especialmente las madres ${ }^{[1]}$. Durante el período perinatal, las familias se adaptan a sus nuevos roles, y pueden aumentar los niveles de estrés, ansiedad y depresión ${ }^{[1]}$. El período perinatal aumenta el riesgo de enfermedades psiquiátricas y los riesgos asociados a morbimortalidad materno-infantil ${ }^{[1]}$.

La depresión perinatal (DPN), de acuerdo con la quinta edición del Manual Diagnóstico y Estadístico de los Trastornos Mentales (DSM-5, por sus siglas en inglés), es un episodio depresivo mayor con inicio en el período perinatal, es decir, se presenta durante el embarazo o en las primeras cuatro semanas después del parto ${ }^{[2]}$. En Perú, Masías y Arias encontraron una prevalencia de 14,15\% de depresión posparto en una posta de la ciudad de Arequipa, utilizando la Escala de Depresión Posnatal de Edimburgo ${ }^{[3]}$. Sin embargo, en un estudio realizado en el Hospital Nacional Dos de Mayo (Lima) y otro en el Hospital Nacional Cayetano Heredia estimaron una prevalencia de depresión posparto de $34 \%$ y $31,4 \%$, respectivamente ${ }^{[3]}$.

Por otro lado, la expansión de manera acelerada de la enfermedad por coronavirus 2019 (COVID-19) en el mundo ha producido un impacto significativo en la salud pública global ${ }^{[4]}$. En el Perú, el gobierno emitió el Decreto Supremo 044-2020$\mathrm{PCM}{ }^{[5]}$ que dispuso la suspensión de las actividades laborales en el sector público y privado, aislamiento social obligatorio y cierre de fronteras por un periodo de 15 días calendario, con la intención de frenar la expansión del COVID-19. En ese contexto, vivir bajo las condiciones de aislamiento social puede tener un impacto en la salud de los peruanos aumentando el riesgo de estrés y ansiedad ${ }^{[6]}$. Asimismo, las emergencias de salud pública a nivel mundial como la pandemia del COVID-19 producida por el SARS-CoV-2, podrían llevar a situaciones de estrés extremo, ansiedad y depresión en mujeres en etapa perinatal ${ }^{[7]}$. Una publicación de Fakiri y Simbar de Irán (disponible en línea el 16 de marzo 2020) documentó que las gestantes, durante la pandemia del COVID-19, manifestaron diversas preocupaciones como no acudir a sus controles prenatales por el riesgo percibido de estar expuestas al COVID-19. Además, los autores encontraron que, debido al estrés y la ansiedad, muchas madres reportaron uso constante (o incluso sobreuso) de productos basados en hipoclorito de sodio y alcohol que podrían causar intoxicación ${ }^{\left[{ }^{[8]}\right.}$ o incluso quemaduras.

La DPN puede ocasionar a la madre y al entorno familiar múltiples consecuencias negativas. En China, Wu et al. ${ }^{[7]}$ encontraron en un estudio transversal multicéntrico en 25 hospitales en 10 provincias, que después de la declaración del brote del COVID-19, se registró un incremento en la prevalencia de DPN incluso con cifras que llegaron hasta un $34,2 \%$ del 5 de febrero al 9 de febrero de 2020. Los autores identificaron que la población más vulnerable incluyó aquellas con las siguientes características: mujeres menores de 35 años con bajo peso, con trabajo a tiempo completo, de clase media, primíparas, y con poca actividad física ${ }^{[7]}$. Además reportaron que las mujeres con un riesgo normal o incluso con depresión leve en general se volvieron más vulnerables a presentar DPN después del brote ${ }^{[7]}$.

En ese sentido, y considerando los riesgos de la infección por el COVID-19 en las mujeres embarazadas ${ }^{[8]}$, cada vez más se reconoce la necesidad de apoyar a las mujeres desde el embarazo hasta el puerperio, en el uso apropiado del Internet y las redes sociales para obtener información de calidad, apoyo y soporte para expresar sus dificultades ${ }^{[9]}$. Así, las redes sociales, bien utilizadas, pueden constituir una herramienta valiosa de apoyo para las mujeres en esta etapa de su vida.

Este artículo describe el rol de las redes sociales en Internet como soporte de las gestantes y madres con depresión, particularmente en emergencias de salud pública a nivel mundial como la pandemia del COVID-19.

\section{El COVID-19 y la desinformación en las redes sociales}

En medio de la crisis de salud pública debido al COVID-19 ya se han comenzado a sentir los efectos de la desinformación, tanto en Internet como en las redes sociales ${ }^{[10]}$. Urge promover evidencia científica y desarrollar estrategias para combatir la información errónea, noticias falsas y rumores que se empiezan a propagar por las redes sociales ${ }^{[10,11]}$.

Los medios de comunicación deben asumir la responsabilidad de proporcionar información correcta y desarrollar material comprensible para los ciudadanos ${ }^{[11]}$. Los periodistas tienen un papel importante en la comunicación efectiva de riesgos y deben reconocer que titulares impactantes pero inexactos y algunas veces engañosos agitan al público y causan miedo, afectando de manera perjudicial a la salud pública ${ }^{[11]}$. Los profesionales de la salud deben cooperar con los medios de comunicación y ayudar a diferenciar lo que se conoce y se desconoce ${ }^{[11]}$.

La comunicación efectiva de riesgos no solo contribuirá a disminuir el riesgo de conductas inapropiadas, como visitar innecesariamente los establecimientos de salud, o saturar las líneas del Ministerio de Salud ${ }^{[12]}$, sino que contribuirá a la disminución de las noticias falsas ${ }^{[11]}$.

\section{Las redes sociales como soporte social para los padres}

El término «redes sociales» en Internet se asocia comúnmente con servicios populares en la web como Facebook, Instagram, Twitter y YouTube; sin embargo, también puede abarcar una gama de otros servicios en línea, como blogs y tableros de mensajes ${ }^{[13]}$. Las personas acceden a Internet a través de una variedad de dispositivos que incluyen computadoras portátiles, teléfonos inteligentes, entre otros. En el Perú, Facebook es la red social más popular en Internet ${ }^{[14]}$.

Las redes sociales, especialmente cuando se acceden desde tecnologías móviles, como los teléfonos inteligentes, ofrecen 
una mayor flexibilidad para obtener beneficios del soporte social en línea y están activas en todo momento, durante los siete días de la semana ${ }^{[15]}$. Debido a su portabilidad y facilidad de uso, las tecnologías móviles son particularmente útiles para ayudar a los padres de niños pequeños a mantenerse conectados ${ }^{[15]}$. Las redes sociales pueden contribuir a la salud y al bienestar al facilitar la interacción social, construir comunidades, normalizar el comportamiento de búsqueda de ayuda, inspirar cambios saludables en el estilo de vida y ayudar a la investigación médica ${ }^{[15]}$. A través de las redes, las mujeres en etapa perinatal pueden acceder a material claro, sencillo y directo sobre el COVID-19 y el embarazo, incluso a través de infografías. Una guía muy amigable que puede tomarse como referencia y que explica de manera clara y directa información sobre el COVID-19 y su relación con el embarazo es desarrollada por el Hospital de Sant Joan de Déu Barcelona en España ${ }^{[16]}$.

Las diversas formas de apoyo social en línea tienen varios beneficios, incluida la capacidad de acceder a información, comunicación e interacción, todo sin tener que abandonar el hogar ${ }^{[17]}$. El acceso a información y experiencias compartidas pueden ser particularmente enriquecedoras durante los períodos posteriores al nacimiento y en la primera infancia, particularmente para las madres que descubren que su red física inmediata no satisface sus necesidades de información o apoyo en el hogar ${ }^{[15]}$.

Madge y O'Connor en su estudio sobre «madres cibernéticas» argumentan que Internet proporciona fuentes de información alternativas que aumentan el sentido real de empoderamiento y ayudan a mejorar algunos de los desafíos como convertirse en madre ${ }^{[18]}$. Dicho acceso a la información y el apoyo en la web puede empoderar a las mujeres al ayudarlas a identificar sus propias necesidades, controlar el estrés, la fatiga y así, responder de manera más efectiva en momentos de estrés ${ }^{[19,20]}$.

Estudios previos encontraron que una sensación de comunicación a distancia, e incluso en ocasiones a través de una percepción de anonimato (incluso cuando el anonimato no siempre esté asegurado), puede alentar a las mujeres a compartir temas que de otra manera no estarían cómodas compartiendo con su propia familia y amigos ${ }^{[21]}$. Incluso dicho acceso a las redes sociales en Internet ofrece la oportunidad de desarrollar una poderosa narrativa que sintoniza con otras mujeres que tienen experiencias similares y facilita la creación de lo que Johnson llama espacios "públicos de maternidad íntima» ${ }^{[22] .}$

\section{Desafíos de las redes sociales en Internet como soporte contra la depresión perinatal}

¿Qué factores podrían influir en las madres para no buscar soporte contra la DPN en sus redes sociales? A continuación, revisaremos la situación por la que atraviesan muchas madres a nivel global.

En un estudio realizado por Barkin et al. ${ }^{[23]}$ en Estados Unidos observaron que dentro de las barreras para acceder a soporte social incluían la poca disponibilidad de cuidado infantil confiable, costos elevados para el cuidado infantil, alta demanda del cuidado infantil, las prioridades cambiantes, la mudanza familiar, entre otros. Asimismo, existen madres que son reacias a solicitar apoyo debido al miedo de ser juzgadas al pedir ayuda, a sentirse como una carga, a una percepción a la crítica, y al orgullo ${ }^{[23]}$. Negron et al. hallaron de manera similar en mujeres de diversas etnias en Nueva York (Estados Unidos) que las actitudes personales y las normas culturales, así como las expectativas de apoyo percibidas, la disponibilidad de apoyo y las relaciones con la pareja y con los miembros de la familia pueden obstaculizar la posibilidad de que una madre solicite apoyo ${ }^{[20]}$.

La falta de voluntad de las madres para acceder al apoyo social puede exacerbar las dificultades existentes asociadas con la nueva paternidad. Mauthner ${ }^{[24]}$ encontró que el miedo y la inseguridad desempeñaban un rol clave en las mujeres que abandonaban el apoyo social. Esto debido a sus creencias ya que perciben que no están cumpliendo con las normas sociales sobre el rol de la maternidad. Estos resultados sugieren que no sólo se requiere tener acceso al soporte real o virtual para que el apoyo social se manifieste. Las madres también necesitan espacios confiables en los que se sientan cómodas para compartir sus preocupaciones o inquietudes sin temor a ser juzgadas o censuradas, y para encontrarse y relacionarse con otras mujeres que han tenido experiencias similares ${ }^{[15]}$. Sin embargo, esto no se logra fácilmente, ya que las mujeres a menudo necesitan acceder a una amplia gama de experiencias sobre la maternidad para darse cuenta de ello, lo cual no siempre es accesible a través de las redes sociales ${ }^{[15]}$. Asimismo, la falta de espacios físicos para reunirse con otras madres, la falta de horarios para interactuar con otras madres e incluso la falta de contacto o espacios de conexión con otras madres se convierten en factores que suman a la lista de desafíos que las mujeres enfrentan en este proceso ${ }^{[24]}$.

Es importante destacar que un desafío constante es discriminar eficientemente la información que encontramos en Internet. Carrilo-Larco et al. ${ }^{[25]}$ encontraron que el $37,4 \%$ de las páginas web en español que contenían información sobre los síntomas del embarazo eran de mala calidad. Los autores recomiendan el desarrollo de guías o intervenciones educativas que enseñen a como evaluar páginas web en relación a la calidad de la información ${ }^{[25]}$. Para ello, los autores recomiendan a los pacientes y público en general utilizar la nemotécnica PRUEBA para evaluar la calidad de la información relacionada a la salud en Internet (Propósito, Responsable, Utilidad de la Información, Enlaces, Buscar el compromiso de confidencialidad y Actualización) ${ }^{[25]}$.

\section{Motivaciones para el uso de las redes sociales}

Si bien las redes sociales ofrecen una serie de beneficios, es importante discutir las motivaciones que subyacen en el uso de las redes sociales. En un estudio realizado por Holtz et al. ${ }^{[26]}$ encontraron que las encuestadas percibieron ventajas 
de pertenecer al grupo de Facebook denominado "Ask the Chicks» (Pregunta a las Chicas, por su traducción al español) sobre otras formas de soporte en línea, ya que ofrecía obtener información inmediata sobre ciertos temas sobre la maternidad y la crianza de los hijos, consejos confiables (debido a la experiencia vivida), e interacción social. Los autores concluyen que contar con este tipo de herramienta de apoyo social es importante ya que se ha encontrado que el apoyo social reduce los niveles de estrés, lo que puede mejorar la salud general y la calidad de vida.

Bartholomew et al., también centrándose en los nuevos padres, examinaron el rol que pueden desempeñar las redes sociales en la construcción del capital social, argumentando que durante los días agitados y ocupados que acompaña a la nueva paternidad, Facebook puede ser un medio particularmente importante para las madres ya que permite construir y mantener el capital social para ellas mismas, sus hijos y sus familias ${ }^{[27]}$.

Otras motivaciones para usar las redes sociales es su poca complejidad de uso ya que muchas personas ya tienen una cuenta en las redes sociales y la usan como parte de sus actividades cotidianas ${ }^{[15]}$. Bartholomew et al. sugieren que el uso de Facebook no sólo se mantiene durante el proceso de transición a la paternidad, sino que incluso en muchos casos se incrementa ${ }^{[27]}$. Las nuevas madres pueden buscar información, unirse a grupos que le interesen, o seguir cuentas ya presentes en esa plataforma de redes sociales, integrando así la actividad en sus rutinas diarias. Esto contrasta con otras formas de apoyo social en línea, como los foros de discusión, ya que a menudo requieren que el usuario cree y mantenga una cuenta separada especialmente para este propósito ${ }^{[26]}$.

Si bien todos los estudios descritos anteriormente son indicadores útiles de por qué y cómo los nuevos padres usan las redes sociales, es importante tener cuidado con la generalización de los usuarios de las redes sociales ${ }^{[15]}$. El uso de las redes sociales como Facebook puede diferir según una serie de factores sociales, económicos y culturares, incluyendo rasgos de personalidad ${ }^{[15]}$.

Las redes sociales también pueden ser un vehículo para reclutar a participantes en intervenciones para prevenir DPN utilizando las redes sociales ${ }^{[28]}$. Haga et al. reportaron los resultados de una intervención automatizada basada en la web denominada "Mamma Mia» en 1342 gestantes noruegas ${ }^{[28]}$. La intervención consistió en tres fases con 44 sesiones durante un período de 11.5 meses. Todas las sesiones incluyeron temas específicos de utilidad durante el período perinatal. La intervención se implementó usando el correo electrónico y a través de páginas web interactivas, combinando texto, imágenes, y archivos de audio pregrabados. Una demostración de esta intervención puede ser accedida desde la siguiente web: http://smarturl. it/psych_med. Se encontró que las madres que recibieron la intervención mostraron menos síntomas depresivos que las participantes en el grupo control.
Asimismo, la información de las redes sociales se puede utilizar para realizar investigaciones sobre DPN. De Chourdhury et al. [29] analizaron datos proporcionados voluntariamente por 165 madres que habían dado a luz y analizaron los mensajes en redes publicados a través de sus muros, fotos, videos, y enlaces incluyendo los "likes» y los comentarios; utilizando modelos estadísticos. Los autores encontraron que a mayor aislamiento social y a menor disponibilidad de capital social en Facebook son los mejores predictores de depresión posparto en las madres [29]. Esta situación puede presentarse en períodos de aislamiento social obligatorio, como es el caso del Perú en épocas de la pandemia del COVID-19. En ese sentido, las herramientas de inteligencia artificial pueden ayudarnos a predecir depresión posparto a través de la información ingresada a través de las redes $^{[30]}$, lo que permitiría desarrollar intervenciones oportunas incluso a través de las redes sociales y evitar complicaciones ${ }^{[31]}$. Estas herramientas de inteligencia artificial pueden estar vinculadas con «chatbots» ${ }^{[32]}$.

\section{Reflexiones finales y recomendaciones}

En esta emergencia de aislamiento social es necesario que el Ministerio de Salud implemente líneas directas de atención psicológica para mujeres en etapa perinatal y soporte en línea a través de Internet (incluyendo la teleorientación psicológica a distancia) en tiempo real para brindar soporte emocional y recomendaciones eficientes y efectivas, incluyendo información a través de las redes sociales como Facebook o Instagram. Asimismo, es importante considerar la implementación de asistentes virtuales o "chatbots» que logren responder las preguntas más frecuentes de las mujeres en etapa perinatal.

Otra propuesta para el soporte emocional a través de las redes sociales debe incluir la participación de "pares», es decir, mujeres que han experimentado DPN, voluntarias e incluso promotoras sociales que podrían proporcionar este tipo de apoyo virtual.

En esta línea, el rol de las universidades es clave, y debería involucrar a sus docentes, investigadores e incluso a estudiantes capacitados quienes, de manera articulada y coordinada, podrían complementar o sumarse a brindar este soporte y apoyo a través de las redes sociales.

Finalmente, es fundamental educary sensibilizar adecuadamente a las mujeres en etapa perinatal, en alfabetización digital en salud que incluya el uso eficiente de las redes en Internet como soporte socioemocional y la evaluación de la calidad de información que se encuentra disponible en las diferentes plataformas del Internet para una adecuada toma de decisiones respecto a su salud y a la de su hijo. Es relevante además contar con material explícito y amigable a través de infografías, videos cortos, etc. sobre las recomendaciones y pautas a seguir en estos casos, para ser distribuidos masivamente en las redes sociales. La Tabla 1 sintetiza los puntos más resaltantes del manuscrito. 
Tabla 1. Puntos más resaltantes del manuscrito.

- La depresión perinatal puede ocasionar a la madre, su bebé y al entorno familiar múltiples consecuencias negativas.

- Las redes sociales, bien utilizadas, pueden constituir una herramienta valiosa de apoyo para las mujeres en esta etapa de su vida.

- Las redes sociales pueden contribuir a la salud y al bienestar al facilitar la interacción social, construir comunidades, normalizar el comportamiento de búsqueda de ayuda, inspirar cambios saludables en el estilo de vida y ayudar a la investigación médica.

- Es fundamental educar y sensibilizar adecuadamente a las mujeres en etapa perinatal en alfabetización digital en salud que incluya el uso eficiente de las redes en Internet como soporte socioemocional y la evaluación de la calidad de información que se encuentra disponible en las diferentes plataformas del Internet para una adecuada toma de decisiones respecto a su salud y a la de su bebé.

- Es relevante además contar con material explícito y amigable a través de infografías, videos cortos, etc. sobre las recomendaciones y pautas a seguir en estos casos, para ser distribuidos masivamente en las redes sociales.

Contribución de los autores: FRBS, WRPA y EEP contribuyeron con la concepción y diseño del trabajo, análisis e interpretación de los resultados, la redacción del manuscrito, revisión crítica y aprobaron la versión final a publicar.

Potenciales conflictos de interés: Los autores declaran no tener conflictos de intereses.

Fuentes de financiamiento: Autofinanciado.

\section{ORCID:}

Fredy R. Bermejo-Sánchez, https://orcid.org/0000-0002-5213-2318 William R. Peña-Ayudante, https://orcid.org/0000-0002-9308-0411 Elizabeth Espinoza-Portilla, https://orcid.org/0000-0002-5068-5357

\section{REFERENCIAS BIBLIOGRÁFICAS}

1. Wisner KL, Miller ES, Tandon D. Attention to Prevention-Can We Stop Perinatal Depression Before It Starts? JAMA Psychiatry. 2019;76(4):355-6. doi: 10.1001/jamapsychiatry.2018.4085

2. Gaviria SL, Duque M, Vergel J, Restrepo D. Síntomas depresivos perinatales: prevalencia y factores psicosociales asociados. Rev Colomb Psiquiat. 2017;45(8):3-4. doi: 10.1016/j. rcp.2017.09.002

3. Vega-Dienstmaier JM. Depresión postparto en el Perú. Rev Med Hered. 2018;29(4):207-10. doi: 10.20453/rmh.v29i4.3444

4. Rodriguez-Morales AJ, Sánchez-Duque JA, Hernández Botero S, Pérez-Díaz CE, Villamil-Gómez WE, Méndez CA, et al. Preparación y control de la enfermedad por coronavirus 2019 (COVID-19) en América Latina. Acta Med Peru. 2020;37(1):3-7. doi: 10.35663/ amp.2020.371.909
5. Gobierno de Perú. Decreto Supremo Nº44-2020-PCM, que declara el Estado de Emergencia Nacional, por las graves circunstancias que afectan la vida de la Nación a consecuencia del brote del COVID-19 [Internet]. Lima, Perú; 2020 [citado el 18 de marzo de 2020]. Disponible en: https://busquedas. elperuano.pe/normaslegales/decreto-supremo-que-declaraestado-de-emergencia-nacional-po-decreto-supremo-n-0442020-pcm-1864948-2/

6. Contagio emocional: Manejo del estrés y la ansiedad en tiempos del Covid-1 [Internet]. Lima, Perú: Gestión; 2020 [citado el 18 de marzo de 2020]. Disponible en: https://gestion.pe/tendencias/ contagio-emocional-manejo-del-estres-y-la-ansiedad-en-tiemposdel-covid-19-noticia/

7. Wu Y-T, Zhang C, Liu H, Duan C-C, Li C, Fan J-X, et al. Perinatal Depression of Women Along with 2019 Novel Coronavirus Breakout in China. Social Science Research Network (SSRN). 2020. doi: 10.2139/ssrn.3539359

8. Rashidi Fakari F, Simbar M. Coronavirus Pandemic and Worries during Pregnancy; a Letter to Editor. Arch Acad Emerg Med. 2020;8(1):e21.

9. Archer C, Kao K-T. Mother, baby and Facebook make three: does social media provide social support for new mothers? Media International Australia. 2018;168(1):122-39. doi: 10.1177/1329878X18783016

10. Mian A, Khan S. Coronavirus: the spread of misinformation. BMC Med. 2020;18(1):89. doi: 10.1186/s12916-020-01556-3

11. Shimizu K. 2019-nCoV, fake news, and racism. Lancet. 2020;395(10225):685-6. doi: 10.1016/S0140-6736(20)30357-3

12. Coronavirus en Perú: línea 113 colapsó por llamadas falsas [Internet]. Lima, Perú: El Comercio; 2020 [citado el 18 de marzo de 2020]. Disponible en: https://elcomercio.pe/videos/pais/ coronavirus-en-peru-linea-113-colapso-por-llamadas-falsas-videovideos-noticia/

13. Wright EM, Matthai MT, Meyer E. The Influence of Social Media on Intrapartum Decision Making: A Scoping Review. J Perinat Neonatal Nurs. 2019;33(4):291-300. doi: 10.1097/JPN.0000000000000377

14. Facebook es la red social favorita de los internautas peruanos [Internet]. Lima, Perú: El Peruano; 2019 [citado el 18 de marzo de 2020]. Disponible en: https://elperuano.pe/noticia-facebook-esred-social-favorita-de-internautas-peruanos-82372.aspx

15. Moon RY, Mathews A, Oden R, Carlin R. Mothers' Perceptions of the Internet and Social Media as Sources of Parenting and Health Information: Qualitative Study. J Med Internet Res. 2019;21(7):e14289. doi: 10.2196/14289

16. Hospital Sant Joan de Déu Barcelona. Coronavirus y embarazo, información para pacientes durante la gestación, el parto y el posparto [Internet]. España, Barcelona: Hospital Sant Joan de Déu Barcelona; 2020 [citado el 18 de marzo de 2020]. Disponible en: https://www. sjdhospitalbarcelona.org/es/coronavirus-embarazo-informacionfiable-para-pacientes-durante-gestacion-parto-posparto

17. Baker B, Yang I. Social media as social support in pregnancy and the postpartum. Sex Reprod Healthc. 2018;17:31-4. doi: 10.1016/j. srhc.2018.05.003

18. Madge $\mathrm{C}, \mathrm{O}^{\prime}$ Connor $\mathrm{H}$. Parenting gone wired: empowerment of new mothers on the internet? Soc Cult Geogr. 2006;7(2):199-220. doi: $10.1080 / 14649360600600528$

19. Chau V, Giallo R. The relationship between parental fatigue, parenting self-efficacy and behaviour: implications for supporting parents in the early parenting period. Child Care Health Dev. 2015;41(4):626-33. doi: 10.1111/cch.12205

20. Negron R, Martin A, Almog M, Balbierz A, Howell EA. Social support during the postpartum period: mothers' views on needs, 
expectations, and mobilization of support. Matern Child Health J. 2013;17(4):616-23. doi: 10.1007/s10995-012-1037-4

21. Buultjens M, Robinson P, Milgrom J. Online resources for new mothers: opportunities and challenges for perinatal health professionals. J Perinat Educ. 2012;21(2):99-111. doi: 10.1891/1058-1243.21.2.99

22. Johnson SA. 'Intimate mothering publics': comparing face-to-face support groups and Internet use for women seeking information and advice in the transition to first-time motherhood. Cult Health Sex. 2015;17(2):237-51. doi: 10.1080/13691058.2014.968807

23. Barkin JL, Bloch JR, Hawkins KC, Thomas TS. Barriers to optimal social support in the postpartum period. J Obstet Gynecol Neonatal Nurs. 2014;43(4):445-54. doi: 10.1111/1552-6909.12463

24. Mauthner N. Postnatal depression: The significance of social contacts between mothers. Women Stud Int Forum. 1995;18(3):311-23. doi: 10.1016/0277-5395(95)80075-Z

25. Carrillo-Larco RM, Pérez-Lu JE, Alvarado-Vásquez E, Curioso WH. Evaluation of the quality of information about pregnancy found in webpages according to the Peruvian guidelines. Rev Peru Med Exp Salud Publica. 2012;29(1):76-81. doi: 10.1590/s172646342012000100011

26. Holtz B, Smock A, Reyes-Gastelum D. Connected Motherhood: Social Support for Moms and Moms-to-Be on Facebook. Telemed J E Health. 2015;21(5):415-21. doi: 10.1089/tmj.2014.0118
27. Bartholomew MK, Schoppe-Sullivan SJ, Glassman M, Kamp Dush CM, Sullivan JM. New Parents' Facebook Use at the Transition to Parenthood. Fam Relat. 2012;61(3):455-69. doi: 10.1111/j.17413729.2012.00708.x

28. Haga SM, Drozd F, Lisøy C, Wentzel-Larsen T, Slinning K. Mamma Mia - A randomized controlled trial of an internet-based intervention for perinatal depression. Psychol Med. 2019;49(11):1850-8. doi: $10.1017 /$ S0033291718002544

29. De Chourdhury M, Counts S, Horvitz EJ, Hoff A. Characterizing and predicting postpartum depression from shared Facebook data. Baltimore, Maryland: Association for Computing Machinery; 2014. p. 626-38. doi: $10.1145 / 2531602.2531675$

30. Farima I, Ud Din Abbasi B, Khan S, Al-Saeed M, Farroq Ahmad H, Mumtaz R. Prediction of postpartum depression using machine learning techniques from social media text. Expert Systems. 2019;36(4). doi: 10.1111/exsy.12409

31. Guntuku SC, Yaden DB, Kern ML, Ungar LH, Eichstaedt JC. Detecting depression and mental illness on social media: an integrative review. Curr Opin Behav Sci 2017;18:43-9. doi: 10.1016/j. cobeha.2017.07.005

32. Powell J. Trust Me, I'm a Chatbot: How Artificial Intelligence in Health Care Fails the Turing Test. J Med Internet Res. 2019;21(10):e16222. doi: $10.2196 / 16222$ 